\title{
Growth anisotropy and self-shadowing: A model for the development of in-plane texture during polycrystalline thin-film growth
}

\author{
O. P. Karpenko, a) J. C. Bilello, and S. M. Yalisove \\ Department of Materials Science and Engineering, University of Michigan, 2300 Hayward Street, \\ Ann Arbor, Michigan 48109-2136
}

(Received 5 September 1996; accepted for publication 22 March 1997)

\begin{abstract}
The development of a preferred crystallographic orientation in the plane of growth, an in-plane texture, is addressed in a model that incorporates anisotropic growth rates of a material and self-shadowing. Most crystalline materials exhibit fast growth along certain crystallographic directions and slow growth along others. This crystallographic growth anisotropy, which may be due to differences in surface free energy and surface diffusion, leads to the evolution of specific grain shapes in a material. In addition, self-shadowing due to an obliquely incident deposition flux leads to a variation in in-plane grain growth rates, where the "fast" growth direction is normal to the plane defined by the substrate normal and the incident flux direction. This geometric growth anisotropy leads to the formation of elongated grains in the plane of growth. Neither growth anisotropy alone can explain the development of an in-plane texture during polycrystalline thin-film growth. However, whenever both are present (i.e., oblique incidence deposition of anisotropic materials), an in-plane texture will develop. Grains that have "fast" crystallographic growth directions aligned with the "fast" geometric growth direction overgrow grains that do not exhibit this alignment. Furthermore, the rate of texturing increases with the degree of each anisotropy. This model was used to simulate in-plane texturing during thin-film deposition. The simulation results are in excellent quantitative agreement with recent experimental results concerning the development of in-plane texture in sputter deposited Mo films. (C) 1997 American Institute of Physics.
\end{abstract} [S0021-8979(97)01413-8]

\section{INTRODUCTION}

Several models have been proposed to explain the development of in-plane texture, a preferred crystallographic orientation in the plane of growth, during thin-film deposition. ${ }^{1-3}$ Most of these models have focused on the effects of an energetic beam or flux, oriented at an oblique angle of incidence relative to the film surface, to explain the evolution of a preferred in-plane crystallographic orientation. The primary mechanisms invoked by these models include preferential resputtering (backsputtering) of unfavorably oriented (high surface energy) planes, ${ }^{1,2}$ ion channeling along close-packed crystallographic directions, ${ }^{1}$ and beam-induced renucleation/recrystallization of grains with favorable orientations (low surface energy). ${ }^{2,3}$ Experimental evidence suggests that these mechanisms do play important roles in the formation of in-plane textures for deposition schemes that involve energetic beams and fluxes (energies of order 100 $\mathrm{eV}$ ) oriented at oblique angles relative to the film surface. However, these mechanisms may not be as important to the development of in-plane textures in situations where the average flux energy is low (of order $1 \mathrm{eV}$ ) and the angular spread in the flux is large, as in the case of sputter deposition at low temperatures. ${ }^{4}$

In-plane textures are known to form in sputter deposited films where the deposition flux is obliquely incident relative to the film surface. ${ }^{5-8}$ Moreover, in-plane textures do not form in films deposited at a normal flux incidence. ${ }^{6}$ These

\footnotetext{
${ }^{a}$ Present address: INTEL Corporation, M/S RN2-35, 2200 Mission College Blvd., Santa Clara, CA 95052.
}

data suggest that oblique flux incidence and self-shadowing play an important role in the development of in-plane texture for sputter deposited films. In this manuscript, a model, which incorporates the effects of self-shadowing and anisotropic growth of a material, is used to explain the phenomenon of in-plane texturing during polycrystalline thin-film growth.

\section{TEXTURING MODEL}

\section{A. Self-shadowing}

The concept of self-shadowing has long been used to explain the development of thin-film growth morphologies. ${ }^{9,10}$ The basic idea behind self-shadowing is concerned with the probability that an atom from the flux will become attached to, or captured by, an atom on a surface. ${ }^{9}$ In order for this to occur, the atoms from the flux must pass close enough to an atom on the surface to become attached. The maximum distance at which a flux atom can still attach to an atom on the surface is called the capture length. The capture length is defined here as two atomic radii, $2 r$ (i.e., only flux atoms that can touch the surface atom will attach). In order to illustrate self-shadowing, it is useful to imagine a cross-sectional view of an atom on a surface where both the flux direction, $\hat{J}$, and the surface normal, $\hat{n}$, lie in the plane of the page [see Figs. 1(b) and 1(c)]. For a single atom on a film surface, the maximum total capture length is $4 r$ and occurs when the flux is directed normal to the film surface [see Figs. 1(a) and 1(b)]. However, this capture length decreases when the flux is incident on the surface at some off-normal angle [see Figs. 1(a) and 1(c)]. In 


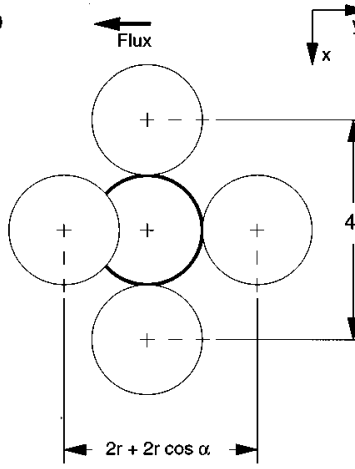

b)
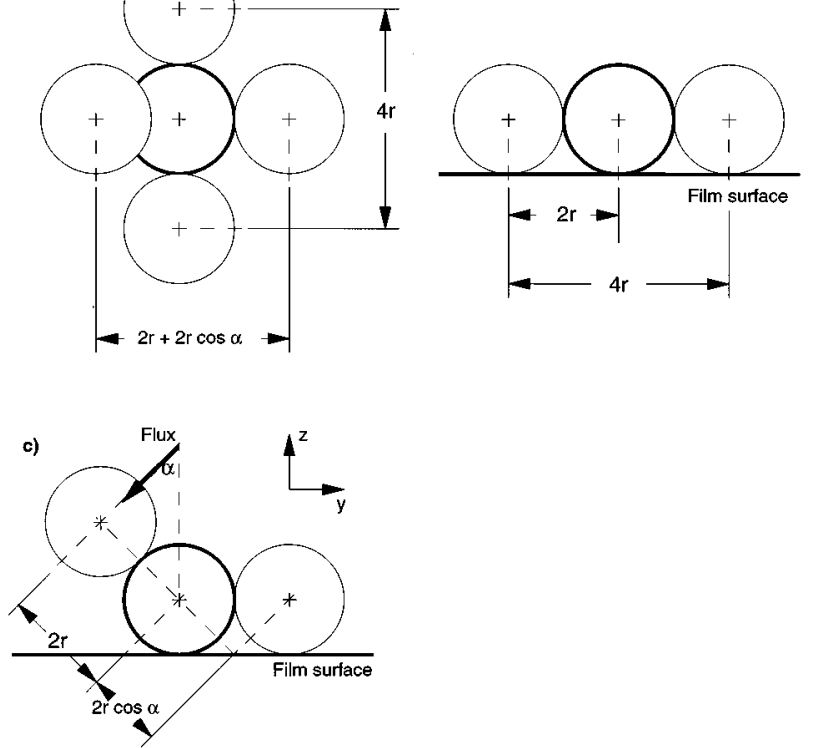

FIG. 1. Schematic diagram illustrating the effect of self-shadowing for a single surface atom. The surface atom is outline in bold. The outermost positions where flux atoms can be captured are marked with a thin outline. The flux, which lies in the $y-z$ plane, is incident on the substrate at an angle $\alpha$ relative to the surface normal. (a) Top view showing both the fast and slow growth directions. (b) Side view showing the fast growth direction. (c) Side view showing the slow growth direction.

this case, an atom on the film surface can capture flux atoms up to a distance $2 r$ in the direction normal to the incident flux (above the film surface), and a distance $2 r$ at an oblique angle relative to the incident flux direction (along the film surface). The total capture length projected by this atom arrangement normal to the incident flux will then be $2 r(1$ $+\cos \alpha$ ), where $\alpha$ is defined as the incident angle of the flux relative to the surface normal.

These arguments can be extended to consider selfshadowing in three dimensions. The specific case treated in this model is growth on a flat surface. The extension to three dimensions is simplified by realizing three things. First, for a flux directed at an oblique angle, $\alpha$, onto a flat surface, one direction along the surface, defined by $(\hat{J} \times \hat{n})$, will have a capture length of $4 r$. Second, for the same flux orientation, one direction along the surface, normal to the first direction and defined by $\hat{n} \times(\hat{J} \times \hat{n})$, will have a capture length of $2 r(\cos \alpha+1)$. Third, these two capture lengths define a capture ellipse [whose major and minor axes are $4 r$ and $2 r(\cos \alpha+1)$, respectively]. This ellipse defines the capture lengths along all directions in the plane of growth. This formulation can be adapted to analyze self-shadowing on a surface with arbitrary topography. Such an analysis is beyond the scope of this model, but the general results should be similar to those presented above.

The most important consequence of this self-shadowing construction concerns the growth rate of islands/grains at the film surface. Clearly, the growth rate of a grain is proportional to the amount of flux it captures. But, it is not apparent

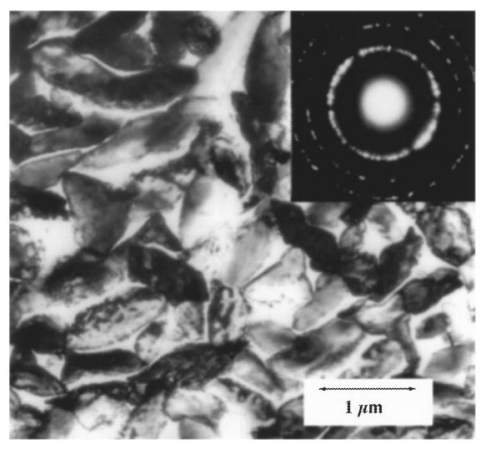

FIG. 2. Plan-view transmission electron microscope micrograph and diffraction pattern showing the grain shapes in a $1 \mu \mathrm{m}$ thick Mo film sputtered onto the native oxide of a $\mathrm{Si}(100)$ wafer in a static normal incidence flux geometry. There are two primary grain shapes; an elliptical grain shape with an aspect ratio between 2 and 3, and a threefold symmetric (pseudopyramidal) grain shape. The diffraction pattern confirms that the film is randomly oriented in the plane of growth (i.e., no in-plane texture).

how flux atoms captured by the grain redistribute on the growth surface to effect grain growth. In the zero-diffusion limit (i.e., growth at low temperatures), atoms captured by the grain cannot redistribute on the growth surface, and grain growth will depend solely on the geometric distribution of flux as dictated by the self-shadowing construction, regardless of the initial grain shape or orientation. At high growth temperatures adatom mobility is high, and grain growth will be dominated by the energetics of the deposited material (i.e., by minimization of total system energy). The case considered here is an intermediate regime where adatom mobility is sufficient to allow the development of distinct grain shapes (i.e., surface diffusion lengths are on the order of the grain dimensions) as shown in Fig. $2{ }^{6}$ and self-shadowing still affects film growth. In order to account for this finite nonzero diffusion, it is convenient to make the following assumptions: (1) Atoms captured by a grain on the film surface can redistribute along the grain surface in a manner consistent with the growth shape of the grain. (2) All atoms captured by the grain contribute to grain growth. (3) Atoms captured at the grain edges contribute to lateral or in-plane grain growth. Based on these assumptions, the amount of flux captured by grain edges can be approximated by $(\cos \alpha+1)$, as in the case of self-shadowing of a single atom on the film surface.

The in-plane growth rates of a grain depend on the amount of flux captured by the grain edge, thus, oblique angle deposition will result in anisotropic in-plane grain growth rates. The slow growth direction lies along the intersection of the film surface and the plane defined by the surface normal and the flux vector. The fast growth direction is normal to this plane $(\hat{J} \times \hat{n})$. In order to better quantify this geometric in-plane growth anisotropy, it is convenient to define a geometric capture efficiency for a grain edge. The geometric capture efficiency, $\nu(\alpha, \chi)$, describes how much flux is captured by a grain edge on the film surface at an arbitrary flux angle, $\alpha$, along an arbitrary in-plane direction, $\chi$, relative to the amount of flux captured from a normally incident flux, $\alpha=0^{\circ}$, along the same in-plane direction. In other words, $\nu(\alpha, \chi)$ is the relative in-plane growth rate of a 
grain. For the fast growth direction, $\chi=0^{\circ}$, the geometric capture efficiency, $\nu_{f}(\alpha)$, is defined as 1 . For the slow growth direction, $\chi=90^{\circ}$, the geometric capture efficiency, $\nu_{s}(\alpha)$, can be expressed with the following integral:

$$
\nu_{s}(\alpha)=\frac{\int_{\alpha_{1}}^{\alpha_{2}} F(\alpha)(\cos \alpha+1)(\cos \alpha)^{2} d \alpha}{\int_{\alpha_{1}}^{\alpha_{2}} F(\alpha) 2(\cos \alpha)^{2} d \alpha},
$$

where $\alpha$, is the angle between the surface normal and the flux direction, $F(\alpha)$, is the angular distribution of the flux, $\cos \alpha+1$, is the geometric dependence for atom capture along a grain edge, $(\cos \alpha)^{2}$, accounts for the $1 / r^{2}$ dependence of the flux on distance from the source, and, $\alpha_{1}$, $\alpha_{2}$, define the angular region over which the substrate "sees" the flux.

It is also assumed that the oblique incidence is a result of moving the substrate an appropriate distance from under the source, and not due to tilting the substrate under the source. For planar magnetron sputtering, the angular distribution of the flux depends on a number of factors (i.e., system geometry, sputtering gas pressure, plasma energy), but can be approximated by $\cos ^{3} \theta$, where $\theta$ is the angle between the source normal and the flux direction. ${ }^{11}$ Using this expression for $F(\alpha)$, Eq. (1) can be simplified.

$$
\nu_{s}(\alpha)=\frac{\int_{\alpha_{1}}^{\alpha_{2}}(\cos \alpha)^{6} d \alpha}{2 \int_{\alpha_{1}}^{\alpha_{2}}(\cos \alpha)^{5} d \alpha}+\frac{1}{2} .
$$

Once $\nu_{f}(\alpha)$ and $\nu_{s}(\alpha)$ are determined, they can then be used to calculate the geometric capture efficiency, or relative growth rate, $\nu(\alpha, \chi)$, along any in-plane direction, $\chi$. As mentioned previously, for a flat surface, the geometric capture efficiency will be equal to the distance from the origin to the edge of an ellipse, where $\nu_{f}(\alpha)$ and $\nu_{s}(\alpha)$ are the major and minor axes, respectively, and $\chi$ is the polar angle.

$$
\nu(\alpha, \chi)=\sqrt{\frac{\nu_{f}^{2}(\alpha) \nu_{s}^{2}(\alpha)}{\nu_{s}^{2}(\alpha) \cos ^{2} \chi+\nu_{f}^{2}(\alpha) \sin ^{2} \chi}} .
$$

In addition to defining the capture ellipse, $\nu_{f}(\alpha)$ and $\nu_{s}(\alpha)$ can be used to define an effective incident flux angle, $\alpha_{\text {eff }}$ for a given geometry.

$$
\alpha_{\text {eff }}=\arccos \left(\frac{\nu_{s}(\alpha)}{\nu_{f}(\alpha)}\right) .
$$

$\alpha_{\text {eff }}$ is a particularly useful quantity, as it fully describes the geometric growth anisotropy due to self-shadowing, the oblique flux, and the angular flux distribution. Calculation of $\alpha_{\text {eff }}$ will allow direct comparison between different deposition geometries based on values of $\alpha_{\text {eff }}$, provided the angular flux distribution can be reasonably approximated.

\section{B. Anisotropic grain growth: Growth shapes}

Another important element in the texturing model is the anisotropic growth of grains in a film. As already mentioned, many materials tend to develop distinct grain (growth) shapes. For example, Fig. 2 shows several different growth shapes that can develop during normal incidence sputter deposition of Mo films. Previous work has shown that the growth shapes of grains can depend on the out-of-plane orientation of the grain. ${ }^{6}$ Furthermore, the prominent features in each grain shape correspond to specific crystallographic directions (i.e., in Mo the "elliptical" grains have a Mo $\langle 100\rangle$ direction aligned with the major axis of the "ellipse"), Although the factors that can influence the final growth shape of the grains are many (i.e., surface energetics, ${ }^{12}$ surface diffusion, ${ }^{13-15}$ impurities, ${ }^{16}$ flux energetics, ${ }^{17}$ and growth temperature $^{6}$ ), we speculate that the growth shape of a grain with a given out-of-plane orientation is most heavily influenced by anisotropic diffusion and mass transport along different crystal surfaces and directions. ${ }^{13-15}$ Previous work studying the surface self-diffusion of refractory metals shows that the activation energy for diffusion varies considerably from surface to surface [e.g., for W surface self-diffusion, $E_{\text {act }}=0.89 \mathrm{eV}$ on $\mathrm{W}(110)$ and $E_{\text {act }}=1.78 \mathrm{eV}$ on W (111)], ${ }^{13,14}$ and that the rate of surface diffusion on a given surface is highly anisotropic. ${ }^{13,14}$ In any event, the grain shapes do provide valuable information about the relative growth rates along different crystallographic directions.

An elliptical grain shape has been chosen for this model to represent Mo grains with (110) out-of-plane textures. Previous work has shown that Mo grains with (110) out-ofplane orientations are nearly elliptical in shape with an aspect ratio of $\sim 2$; Mo $\langle 100\rangle$ and Mo $\langle 110\rangle$ are aligned along the major and minor axes, respectively (see Fig. 2). ${ }^{6,18}$ For this grain shape, the growth velocity, $u$, along any in-plane crystallographic direction, $\psi$, was approximated by the length between the origin (center of mass) of the grain and the grain edge ${ }^{12}$ (effectively, a reverse Wolff construction).

$$
u(\psi)=\sqrt{\frac{u_{110}^{2} u_{100}^{2}}{u_{110}^{2} \cos ^{2} \psi+u_{100}^{2} \sin ^{2} \psi}},
$$

where $\psi$, is the in-plane polar angle, $u\left(0^{\circ}\right)=u_{100}$ is the fast growth direction, $u\left(90^{\circ}\right)=u_{110}$ is the slow growth direction, and $R=u_{100} / u_{110}$ is the aspect ratio of the grain shape.

\section{Grain growth and texturing}

Grain growth was investigated by considering the effect of geometric capture efficiency at a grain edge, $\nu\left(\alpha_{\text {eff, } \chi}\right)$, and anisotropic grain growth rates, $u(\psi)$. For any grain in the film, the growth rate, $g\left(\alpha_{\text {eff, }, \phi}\right)$, along a specific direction, $\psi$, was defined as the product of $u(\psi)$ and $\nu\left(\alpha_{\text {eff }}, \chi\right)$ along that direction. Although the exact relationship between growth rate, $u(\psi)$ and $\nu\left(\alpha_{\text {eff }}, \chi\right)$ is not clear, for the sake of simplicity, the growth rate was taken as a simple multiplicative product of $u(\psi)$ and $\nu\left(\alpha_{\text {eff }}, \chi\right)$. In order to determine this growth rate, it is convenient to define a variable, $\phi$, which defines the angle between $\nu\left(\alpha_{\text {eff }}, 0\right)$, the fast growth direction in the geometry, and $u(0)$, the fast growth direction in the grain. Using $\phi, \psi$ can be rewritten as $\chi+\phi$, and the growth rate, $g\left(\alpha_{\text {eff }}, \chi, \phi\right)$, along any direction can be written as a function of $\phi$ and $\chi$.

$$
g\left(\alpha_{\text {eff }}, \chi, \phi\right)=\nu\left(\alpha_{\text {eff }}, \chi\right) u(\chi+\phi) .
$$

$g\left(\alpha_{\text {eff }}, \chi, \phi\right)$ can be thought of as the product of two probabilities; $\nu\left(\alpha_{\text {eff }}, \chi\right)$ is the probability for flux capture at a grain edge, and $u(\chi+\phi)$ is the probability that an atom cap- 


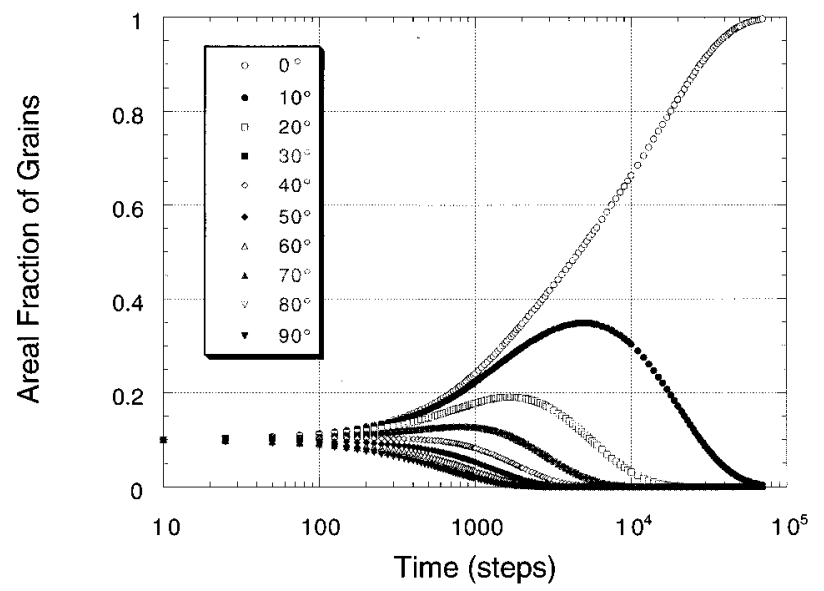

FIG. 3. Plot showing the evolution of areal fractions of grains with various in-plane orientations, as a function of time (growth steps). The data were calculated for an effective incident flux angle of $10^{\circ}$, and for elliptical grains with an aspect ratio of $2.0^{\circ}$ corresponds to the alignment of both fast growth directions (geometric capture and grain growth).

tured at the grain edge will contribute to growth along the direction $\chi+\phi$. The total areal in-plane growth rate, $G\left(\alpha_{\text {eff }}, \phi\right)$, which is dependent on $\phi$, and $\alpha_{\text {eff }}$ (geometric anisotropy), can be determined by integrating $g\left(\alpha_{\text {eff }}, \chi, \phi\right)$ over $\chi$. For convenience, $G\left(\alpha_{\text {eff }}, \phi\right)$ is normalized by $G\left(\alpha_{\text {eff }}, \phi=0\right)$, the growth rate when the fast growth directions are aligned,

$$
G\left(\alpha_{\mathrm{eff}}, \phi\right)=\frac{\int_{0}^{2 \pi} \nu\left(\alpha_{\mathrm{eff}}, \chi\right) u(\chi+\phi) d \chi}{\int_{0}^{2 \pi} \nu\left(\alpha_{\mathrm{eff}}, \chi\right) u(\chi) d \chi} .
$$

Using $G\left(\alpha_{\text {eff }}, \phi\right)$, the evolution of in-plane texture can be investigated as a function of time, $t$, grain orientation, $\phi$, deposition anisotropy (geometry), $\alpha_{\text {eff }}$, and grain shape anisotropy, $R$. First, the growth rates, $G\left(\alpha_{\text {eff }}, \phi\right)$, must be calculated for each value of $\phi$ (due to the symmetry of this system, all growth directions can be represented within the angular range $\left.0^{\circ}-90^{\circ}\right)$. Once each $G\left(\alpha_{\text {eff }}, \phi\right)$ is calculated, the areal fractions of each grain orientation, $f(\phi, t=0)$, can be chosen. In order to approximate a film with a (110) outof-plane texture and a completely random in-plane texture, $f(\phi, t=0)=1 / m$, where $m$ is the number of in-plane grain orientations. These grains can then be allowed to grow by calculating new values for $f(\phi, t)$ at each time step, $t$. For each $t, f(\phi, t)$ is determined by multiplying $f(\phi, t-1)$ by $G\left(\alpha_{\text {eff }}, \phi\right)$, and normalizing by the total grain area at $t$,

$$
f\left(\phi_{i}, t\right)=\frac{f\left(\phi_{i}, t-1\right) G\left(\phi_{i}\right)}{\sum_{i} f\left(\phi_{i}, t-1\right) G\left(\phi_{i}\right)}=\frac{f\left(\phi_{i}, 0\right) G^{t}\left(\phi_{i}\right)}{\sum_{i} f\left(\phi_{i}, 0\right) G^{t}\left(\phi_{i}\right)}
$$

\section{EVOLUTION OF IN-PLANE TEXTURE}

\section{A. Dependence on in-plane grain orientation, $\phi$}

The influence of grain orientation on the evolution of in-plane texture is shown in Fig. 3. The data was generated for a film with ten different grain orientations, $\phi=0^{\circ}-90^{\circ}$ in $10^{\circ}$ increments, and equal initial areal fractions $f\left(\phi_{i}, 0\right)$ $=0.10$. The values for $\alpha_{\text {eff }}$ and $R$ were chosen arbitrarily; $\alpha_{\text {eff }}=10^{\circ}$ and $R=2$. The plot shows that the growth and

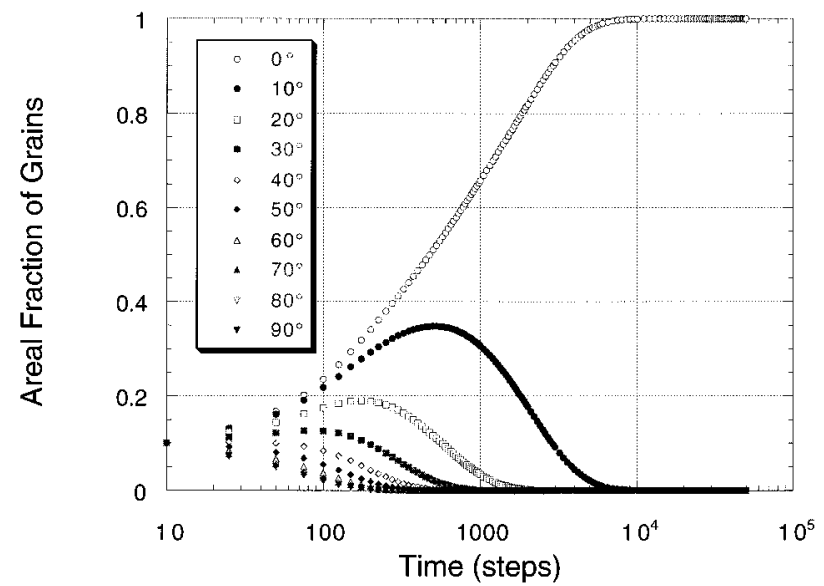

FIG. 4. Same as Fig. 3, except that the effective incident flux angle is $30^{\circ}$.

decay rates of $f\left(\phi_{i}, t\right)$ have a strong dependence on $\phi$, the grain orientation in the plane of growth. The areal fractions, $f\left(\phi_{i}, t\right)$, of grains with large misorientations $\left(\phi \geqslant 50^{\circ}\right)$ decrease immediately from the start of growth, with a decay rate that increases with $\phi$. For grains with smaller misorientations $\left(\phi \leqslant 40^{\circ}\right), f\left(\phi_{i}, t\right)$ initially increases, but then decays at a later time for all orientations, except for $\phi=0^{\circ}$. This decay is delayed to later times for decreasing values of $\phi$. The only grain orientation that survives after long growth times is $\phi=0^{\circ}$. This demonstrates that grains whose fast crystallographic growth directions are aligned with the fast geometric growth direction have a growth advantage over grains of other orientations, and that these aligned grains will determine the final in-plane texture orientation. For the elliptical grains used here, the fast growth direction is $\langle 100\rangle$. These results reproduce experimental results for the development of in-plane texture in sputtered Mo films. ${ }^{5-7}$ Mo films with (110) out-of-plane textures, exhibit an alignment of $\langle 100\rangle$, the fast growth direction, normal to the plane defined by the surface normal and the flux direction, and an alignment of $\langle 110\rangle$, the slow growth direction, along the intersection of this plane with the film surface.

\section{B. Influence of the deposition geometry, $\alpha_{\text {eff }}$}

In most thin-film deposition systems, the flux is not sufficiently well collimated so that it is incident onto the substrate at one discrete angle. Rather, the flux hits the substrate through a range of incident angles, which may vary from $\pm 3^{\circ}$ for molecular beam epitaxy systems to $\pm 30^{\circ}$ for sputtering systems. For this reason, the geometric capture anisotropy of any deposition geometry is best described by an effective incident flux angle, $\alpha_{\text {eff }}$. Hence, it is important to determine the influence of $\alpha_{\text {eff }}$ on the texturing process. Based on the fact that the geometric capture anisotropy increases with $\alpha_{\text {eff }}$, it is expected that increasing $\alpha_{\text {eff }}$ should accelerate the texturing process. This is confirmed by comparison of Figs. 3 and 4. (For Figs. 3 and 4, $\alpha_{\text {eff }}=10^{\circ}$ and $\alpha_{\text {eff }}=30^{\circ}$, respectively, with all other parameters being the same.) Both Figs. 3 and 4 show the same general trends in the evolution of in-plane texture with grain orientation. However, the evolution of texture occurs at a much faster rate in 


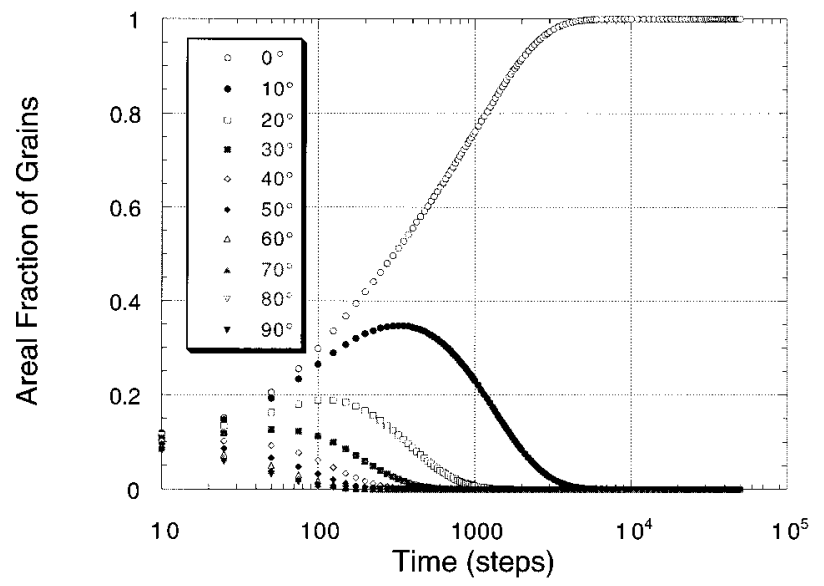

FIG. 5. Same as Fig. 4, except that the elliptical grains have an aspect ratio of 3 .

Fig. 4, as compared to Fig. 3. The value of $\phi(0, t)$ reaches 1 (this corresponds to a completely textured film) after $\sim 7000$ time steps in Fig. 4, and after $\sim 70000$ time steps in Fig. 3; almost an order of magnitude difference in time. These results demonstrate that a greater geometric capture anisotropy dramatically increases the rate at which texture evolves during growth.

\section{Influence of grain shape anisotropy, $R$}

For the elliptical grains, the growth anisotropy of a material is expressed in terms of the aspect ratio, $R$, of the grains. Increasing and decreasing $R$ should have the same effect as increasing and decreasing $\alpha_{\text {eff }}$. Comparison between Figs. 4 and 5 bear this out. (For Figs. 4 and 5, $R=2$ and $R=3$, respectively, with all other parameters being the same.) Both Figs. 4 and 5 show the same general trends in the evolution of in-plane texture with grain orientation, except that the evolution of texture occurs at a slightly faster rate in Fig. 5, as compared to Fig. 4. These results further demonstrate that the degree of anisotropy in the system strongly influences the rate of in-plane texturing during thinfilm growth.

\section{COMPARISON TO EXPERIMENT}

Recent experimental results concerning the evolution of in-plane texture in sputter deposited Mo films provide an ideal test of the model developed here. The key elements that will be compared are: (1) the alignment and morphology of textured grains, and (2) the rate of texture evolution in different deposition geometries.

Experimental results indicate that sputter deposited Mo films tend to form (110) out-of-plane and in-plane textures in deposition geometries with oblique incident flux angles. The Mo grains are nearly elliptical in shape, where the major axis of the ellipse, a Mo $\langle 100\rangle$ direction, is aligned along the direction defined by the cross product of the flux vector and the surface normal. The results from this texturing model reproduce this growth morphology. The grain shapes in the

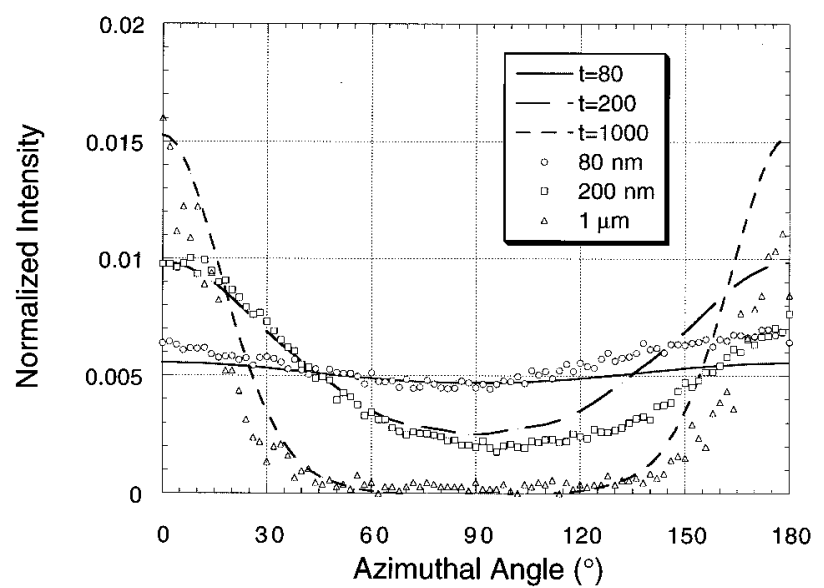

FIG. 6. The plot shows a comparison between the azimuthal distribution of (200) peaks in experimental data collected from sputter deposited Mo films, and calculated diffraction data for an effective incident flux angle of $23^{\circ}$ and elliptical grains with an aspect ratio of 2 . The experimental data, displayed with individual data points, were collected during an azimuthal scan done in a symmetric Bragg grazing incidence x-ray scattering geometry for films 80 $\mathrm{nm}, 200 \mathrm{~nm}$, and $1 \mu \mathrm{m}$ thick. The simulated data, shown as solid lines, correspond to 40, 100, and 500 time steps. Each time step roughly corresponds to $2 \mathrm{~nm}$ of deposited film thickness.

model are also elliptical with a Mo $\langle 100\rangle$ direction along the major axis of the ellipse, aligned with the fast geometric growth direction.

The evolution of texture in sputtered Mo films, as predicted by this model, can be easily compared to experiment by calculating diffraction patterns from the modeling data and comparing them to diffraction patterns collected from sputter deposited Mo films. These diffraction patterns are shown in Fig. 6. Both the real and calculated diffraction patterns show that at small film thicknesses (growth times), the films display a poorly defined in-plane texture. As the film thickness (number of growth steps) is increased, the in-plane texture becomes more defined; the width and intensity of the texture peak decrease and increase, respectively. Comparison between the two sets of diffraction patterns in Fig. 6 suggests that each time step in the model corresponds to $\sim 2 \mathrm{~nm}$ of deposited film thickness.

This model was also used to predict the relative rate of in-plane texturing for several different experimental geometries. The first and simplest geometries that were explored are off-axis oblique incidence geometries. In these deposition geometries, the substrate is held in a stationary position, such that the substrate and source surfaces are parallel and a

TABLE I. Influence of deposition geometry on $\alpha_{\text {eff }}$ (the geometric growth anisotropy).

\begin{tabular}{ccc}
\hline \hline Angular range & Deposition geometry & $\alpha_{\text {eff }}$ \\
\hline $0^{\circ}$ & Static & $0^{\circ}$ \\
$10^{\circ} \pm 5^{\circ}$ & Static & $7.26^{\circ}$ \\
$20^{\circ} \pm 5^{\circ}$ & Static & $14.06^{\circ}$ \\
$30^{\circ} \pm 5^{\circ}$ & Static & $20.89^{\circ}$ \\
$40^{\circ} \pm 5^{\circ}$ & Static & $27.64^{\circ}$ \\
$0^{\circ}-90^{\circ}$ & Dynamic & $16.22^{\circ}$ \\
\hline \hline
\end{tabular}


line drawn between the source and substrate centers is at a fixed angle, $\gamma$, relative to the surface normals. Values of $\alpha_{\text {eff }}$ were calculated for each desired incidence angle, $\gamma$. This was done by integrating expression (2) through an angular range defined $\gamma-5^{\circ}-\gamma+5^{\circ}$. Table I, shows $\alpha_{\text {eff }}$ for $\gamma=0^{\circ}, 10^{\circ}, 20^{\circ}, 30^{\circ}$, and $40^{\circ}$. As expected, $\alpha_{\text {eff }}$ increases with $\gamma$. Consequently, as $\gamma$ increases, so does the rate of in-plane texturing. This is consistent with recent experimental observations. ${ }^{5}$

One other deposition geometry was investigated; a dynamic deposition geometry. In this geometry, the substrate is placed on a rotating carousel and passes under the source once every carousel revolution. In this geometry, the substrate effectively "sees" all angles of incident flux, so the integral in expression (2) is evaluated from $0^{\circ}-90^{\circ}$. The effective incident flux angle, $\alpha_{\text {eff }}$, for this geometry is also listed in Table I. The most interesting feature in Table I is the comparison of $\alpha_{\text {eff }}$ for the off-axis geometries with different values of $\gamma$, to $\alpha_{\text {eff }}$ for the dynamic deposition geometry. Based on the values for $\alpha_{\text {eff }}$, one would predict that the evolution of texture in the dynamic geometry would proceed at a faster rate than in the off-axis geometry with $\gamma \leqslant 20^{\circ}$, but at a substantially slower rate than with $\gamma \geqslant 30^{\circ}$. Experimental results have substantiated both predictions. The micrograph in Fig. 2 shows that no in-plane texture develops for static growth at a normal flux incidence, $\gamma=0^{\circ}$, in agreement with the former predicted behavior.

\section{EXTENSION TO OTHER FACTORS AFFECTING IN-PLANE TEXTURING}

The present work considers the effect of deposition geometry and the anisotropic growth of grains on the evolution of in-plane textures in films with well-defined out-of-plane textures. There are, of course, numerous other factors that can contribute to the development of thin-film textures. These include, but are not limited to, flux energy, ${ }^{17}$ resputtering of the deposited material, ${ }^{1,2,19,20}$ surface topography, ${ }^{21}$ impurities, ${ }^{16}$ and the development of an out-of-plane texture. ${ }^{6,19}$ For the sake of completeness, the effects of an energetic flux and the effect of surface topography on the development of in-plane texture will be addressed.

The effect of an energetic flux on the evolution of thinfilm texture and morphology is important to consider, as some particles incident on the film surface can attain an energy in excess of $\sim 100 \mathrm{eV}$ during sputter deposition. ${ }^{22} \mathrm{~A}$ number of experimental and theoretical studies have investigated resputtering of deposited material during growth. ${ }^{2,19,20}$ Results suggest that resputtering rates are anisotropic and are lowest for resputtering of close-packed (low-energy) crystallographic surfaces. ${ }^{1,11}$ As a consequence, anisotropic resputtering may provide an additional growth advantage to grains that have close-packed planes aligned to the flux direction. ${ }^{1,2}$ Additionally, anisotropic resputtering may suppress the development of surface roughness,$^{23}$ or may facilitate the formation of low-indexed facets along the growth surface. ${ }^{6,17}$ Preliminary experimental results suggest that energetic fluxes do, in fact, reduce surface roughness and favor the formation of faceted surfaces, with facets aligned along the flux direction [see Figs. 7a, 7b, and 7c]. It is noted that the
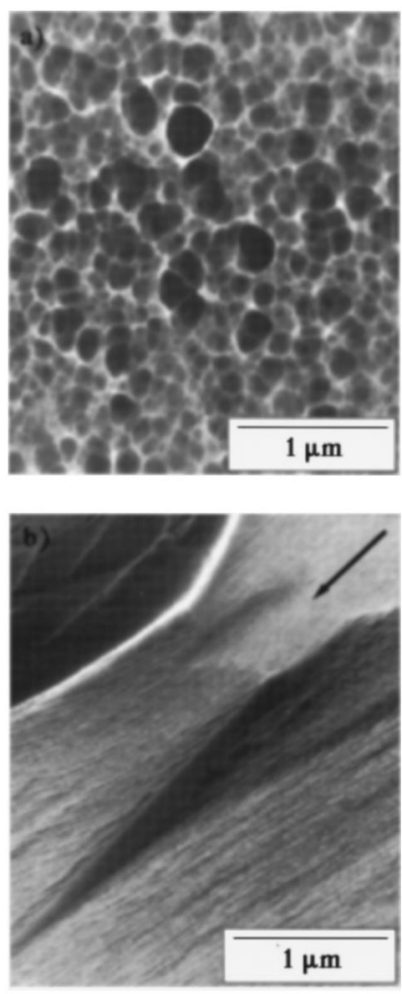

FIG. 7. Plan view scanning electron micrographs of Mo (110) single-crystal surfaces. The surfaces were mechanically polished and chemically etched with a modified Murakami's reagent. (a) Mo (110) surface after polishing and etching. (b) Mo (110) surface after polishing, etching, and ion milling for 30 min with $5 \mathrm{keV} \mathrm{Ar}^{+}$ions, at a beam current of $0.5 \mathrm{~mA}$ from an in-plane Mo $\{112\}$ direction. The flux direction is marked with an arrow in the micrograph.

facet alignment along the flux direction is probably due to the low incidence angle of the ion flux. For greater incident angles, the orientation of the facets is expected to be normal to the flux direction. ${ }^{24}$

Since an energetic flux may facilitate the formation of facets along the growth front of sputter deposited films, it is necessary to consider the effect of a faceted surface topography on the rate of in-plane texturing. To this end, it is sufficient to calculate $\alpha_{\text {eff }}$ for deposition onto a faceted surface and compare it to $\alpha_{\text {eff }}$ for deposition onto a flat surface

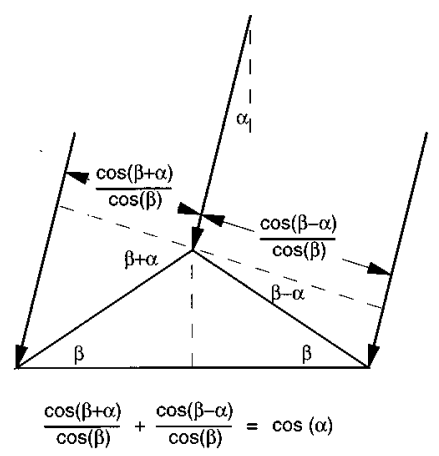

FIG. 8. Schematic showing the geometry of oblique deposition onto a regularly faceted surface. The angle between the flux and each facet is $\beta-\alpha$ and $\beta+\alpha$ for the leading and trailing facets, respectively, where the incident flux angle is $\alpha$ and the facet angle is $\beta$. 
TABLE II. Influence of the facet angle, $\beta$, on $\alpha_{\text {eff }}$ (the geometric growth anisotropy).

\begin{tabular}{ccc}
\hline \hline Facet angle & Deposition geometry & $\alpha_{\text {eff }}$ \\
\hline $0^{\circ}$ & Dynamic & $16.22^{\circ}$ \\
$15^{\circ}$ & Dynamic & $16.08^{\circ}$ \\
$30^{\circ}$ & Dynamic & $15.62^{\circ}$ \\
$45^{\circ}$ & Dynamic & $14.75^{\circ}$ \\
$60^{\circ}$ & Dynamic & $13.23^{\circ}$ \\
\hline \hline
\end{tabular}

for the same deposition geometry. A schematic of a facet pair is shown in Fig. 8. For the sake of simplicity, it is assumed that facets form isosceles triangles, and the facet angle, $\beta$, is measured between each equivalent side and the base of the facet/triangle. A geometric analysis shows that for an incident flux angle $\alpha$ and facet angle $\beta$, the incident angles of the flux onto the leading and trailing facet are $\beta$ $-\alpha$ and $\beta+\alpha$, respectively (see Fig. 8). Since deposition occurs onto both facet surfaces simultaneously, the total capture length of a grain edge along a facet structure is given by $\cos \alpha \cos \beta+1$. This capture length can be substituted for the capture length in expression (2) to determine $\alpha_{\text {eff }}$ for growth onto a faceted surface. Only growth in a dynamic deposition geometry was used for this analysis. Equation (2), with modified capture lengths, was integrated from $0^{\circ}$ to $90^{\circ}$. The effect of facet angle, $\beta$, on $\alpha_{\text {eff }}$ is shown in Table II. Based on this analysis, an increase in $\beta$ effectively reduces $\alpha_{\text {eff }}$ and should retard the rate at which in-plane texture develops. This prediction is currently being investigated in continued experimental studies. $^{25}$

The present model assumes that the film has a welldefined out-of-plane texture at the start of growth. Although most films typically have a random distribution of grains both in-plane and in the growth direction at the start of growth, a strong out-of-plane texture can develop at very low deposited thicknesses. ${ }^{6}$ For this reason, the development of an out-of-plane texture was not addressed. However, a model for the out-of-plane texturing process is being developed.

\section{CONCLUSIONS}

The primary goal of this study was to provide a framework based on physical ideas that can be applied to the analysis of in-plane texturing during low-temperature film deposition. The development of in-plane texture is found to be related to two independent growth anisotropies, geometric capture and grain growth. Specifically, obliquely incident deposition fluxes cause a geometric capture anisotropy at a grain edge through the process of self-shadowing. Similarly, differences in surface free energies and in rates of mass transport along different crystallographic surfaces lead to anisotropic grain growth rates and to the development of anisotropic, faceted, grain shapes (growth shapes). Either one of these anisotropies by itself cannot explain the formation of an in-plane texture. However, the combination of both anisotropies leads to in-plane texturing, where the dominant grain orientation has its fast grain growth direction aligned with the fast geometric growth direction. The rate of in-plane texturing is also affected by the degree of these anisotropies; the more oblique the incident flux angle and the more anisotropic the grain shape, the faster the rate of texturing. These results are in strong agreement with experimental data and point to the conclusion that in-plane texturing is driven primarily by growth anisotropy; the alignment of fast growth directions in the deposited material and in the deposition geometry. Perhaps the most significant implication of this conclusion concerns how the deposition geometry can be used to control the orientation of in-plane textures. Since the in-plane textures result from the alignment of fast growth directions (geometric and crystallographic), careful manipulation of the deposition geometry and the grain shapes (i.e., through the use of multiple sources or surfactants) could result in the formation of specifically tailored film textures. In conclusion, the model developed here has been shown to be capable of predicting specific deposition conditions needed to produce a desired microstructure.

\section{ACKNOWLEDGMENTS}

The authors would like to thank David Eaglesham and David Srolovitz for stimulating discussions, which led to the development of this model. This work was funded by the NSF Contract No. DMR9202176, and by ARPA under Contract No. DAAH04-95-1-0120.

${ }^{1}$ R. M. Bradley, J. M. E. Harper, and D. A. Smith, J. Appl. Phys. 60, 4160 (1986).

${ }^{2}$ N. Sonnenberg, A. S. Longo, M. J. Cima, B. P. Chang, K. G. Ressler, P. C. McIntyre, and Y. P. Liu, J. Appl. Phys. 74, 1027 (1993).

${ }^{3}$ D. Dobrev, Thin Solid Films 92, 41 (1982); G. N. Van Wyk and H. J. Smith, Nucl. Instrum. Methods 170, 433 (1980).

${ }^{4}$ H. Windischmann, Crit. Rev. Solid State Mater. Sci. 17, 547 (1992).

${ }^{5}$ A. K. Malhotra, S. M. Yalisove, and J. C. Bilello, Thin Solid Films 286, 196 (1997).

${ }^{6}$ O. P. Karpenko, J. C. Bilello, and S. M. Yalisove, J. Appl. Phys. 76, 4610 (1994).

${ }^{7}$ O. P. Karpenko, Z. U. Rek, S. M. Yalisove, and J. C. Bilello (unpublished).

${ }^{8}$ D. O. Smith, M. S. Cohen, and G. P. Weiss, J. Appl. Phys. 31, 1755 (1960)

${ }^{9}$ A. G. Dirks and H. J. Leamy, Thin Solid Films 47, 219 (1977).

${ }^{10}$ H. J. Leamy, G. H. Gilmer, and A. J. Dirks, Curr. Top. Mater. Sci. 6, 310 (1980).

${ }^{11}$ A. Wucher and W. Reuter, J. Vac. Sci. Technol. A 6, 2316 (1988); T. Tsuge and S. Esho, J. Appl. Phys. 52, 4391 (1981).

${ }^{12}$ C. Herring, Phys. Rev. 82, 87 (1951).

${ }^{13}$ G. Ehrlich and F. G. Hudda, J. Chem. Phys. 44, 1039 (1966).

${ }^{14}$ W. R. Graham and G. Ehrlich, Surf. Sci. 45, 530 (1974).

${ }^{15}$ G. L. Kellogg, Surf. Sci. Rep. 21, 1 (1994).

${ }^{16}$ M. Horn-von Hoegen, M. Copel, J. C. Tsang, M. C. Reuter, and R. M. Tromp, Phys. Rev. B 50, 10811 (1994).

${ }^{17}$ T. J. Vink and J. B. A. D. van Zon, J. Vac. Sci. Technol. A 9, 124 (1991).

${ }^{18}$ D. P. Adams, L. J. Parfitt, J. C. Bilello, S. M. Yalisove, and Z. U. Rek, Thin Solid Films 266, 52 (1995).

${ }^{19}$ R. W. Smith and D. J. Srolovitz, J. Appl. Phys. 79, 1448 (1996); R. W. Smith, F. Yeng, and D. J. Srolovitz, Mater. Res. Soc. Symp. Proc. 399, 371 (1996).

${ }^{20}$ D. J. Kester and R. Messier, J. Mater. Res. 8, 1938 (1993).

${ }^{21}$ H. Kataoka, J. A. Bain, S. Brennan, and B. M. Clemens, J. Appl. Phys. 73, 7591 (1993)

${ }^{22}$ M. W. Thompson, Philos. Mag. 18, 377 (1968).

${ }^{23}$ N.-E. Lee, G. A. Tomasch, and J. E. Greene, Appl. Phys. Lett. 65, 1 (1994).

${ }^{24}$ E. Chason (private communication).

${ }^{25}$ J. F. Whitacre, O. P. Karpenko, S. M. Yalisove, and J. C. Bilello (unpublished). 